\title{
Image Dehazing Method Based on Multi-scale Feature Fusion
}

\author{
Minghai Yao ${ }^{1, a}$, Qi Miao ${ }^{2, b}$, Qiaohong $\mathrm{Hao}^{2, \mathrm{c}}$ \\ ${ }^{1}$ Department of College Foundation Education, Bohai University, Jinzhou, P.R. China \\ ${ }^{2}$ School of Computer Science and Information Technology, Northeast Normal University,Changchun, P.R. \\ China \\ ayao_ming_hai@163.com, bqim801@nenu.edu.cn, '1031749032@qq.com
}

Keywords: Image dehazing, adaptive clustering, feature fusion, Laplacian pyramid

\begin{abstract}
In recent years, image dehazing has become an active topic in computer vision. This paper presents a novel dehazing method based on adaptive clustering and image fusion for restoring a single image degraded by fog or haze. Firstly, the coarse-scale transmission map is addressed based on adaptive clustering for the haze image. Then, the saturation of haze image is extracted as fine-scale transmission map, which can reflect the scene depth information truly and naturally. Next, the accurate transmission map is generated by using Laplacian pyramid fusion technique. Finally, the haze-free image is restored by solving the image degradation physical model. The effectiveness of the proposed method is evaluated on various real-scene haze images. Experimental results indicate that our proposed method is simpler and more effective compared with several well-known methods, and even achieve better visual results. Specifically, our method is more fast and suitable for real-time requirement.
\end{abstract}

\section{Introduction}

Outdoor images are usually degraded by bad weather conditions, such as haze or fog. The haze or fog weather conditions can easily lead to image contrast reduction, color distortion and detail loss. Therefore, image dehazing has very important theoretical and practical significance. In general, the existing image dehazing methods are divided into two main categories, multiple images based dehazing methods and single image based dehazing methods. The first category methods estimate the scene depths from two or more images of the same scene under different weather conditions [1], or with different degrees of polarizations by routing a polarizing filter attached to the camera [2], or using some prior information [3]. Although these methods can produce good results in certain circumstances, it is impossible and impractical to obtain multiple images from the same scenes or more additional prior information. Thus, the applicability and the real-time performance are limited.

In recent years, dehazing by a single haze image has become a very popular topic in the field of image restoration. Tan et al. [4] proposed an image defogging method based on maximizing image contrast, meanwhile keeping the smoothness of the local airlight for improving image visibility. However, this method leads to over-compensation phenomenon and makes color of recovery image distorted. Fattal et al. [5] obtained the haze-free image using the assumption that local irrelevant between the shading surface of the object and the transmission function. But the dehazing effect of this method is not ideal for heavy haze image. He et al. [6] presented a single image dehazing method based on dark channel prior (DCP). Although this method has good effect for most of the haze images, refining transmission map by the soft matting technique is very time-consuming. Tarel et al. [7] proposed a fast dehazing method using median filter. Nevertheless, this method cannot obtain satisfactory results in the edge region of scene depth jumps. Kim et al. [8] established a cost function via taking into account the loss of contrast and information simultaneously for static image dehazing. Unfortunately, it couldn't effectively remove the haze completely. Zhang et al. [9] proposed a single image visibility enhancement method using low-rank technique and overlap average strategy. The experimental results indicate that this method cannot remove the haze completely. 
This paper presents a fast and effective single dehazing method based on clustering and image fusion techniques. For the sake of reducing block effect in dark channel prior method, the coarse-scale transmission map is derived sufficiently from the minimum channel of the haze image based on adaptive clustering. Meanwhile, the saturation map of haze image is extracted as fine-scale transmission map, which can reflect the scene depth information properly. Then, in order to derive the accurate transmission map, Laplacian pyramid fusion technique is adopted to fuse the two scale transmission maps. Finally, the haze-free image is restored by solving the degradation model. The experimental results show that our proposed method is simple but effective, and can achieve satisfactory dehazing results. Moreover, it can be comparable with several state-of-the-art methods, and even achieve better results and real-time requirement.

\section{Image Degradation Model}

In this paper, we estimate atmospheric light by improving Kim et al.'s method based on quad-tree [8]. Firstly, the haze image is divided into four sub-images. Then, the mean and standard deviation are calculated for each sub-image, and the difference between mean and standard deviation is taken as the score. Next, the sub-image with maximum score will be decomposed iteratively, until the size of sub-image is smaller than a threshold. Finally, instead of the maximum value, the mean value of sub-image with maximum score is chosen as the atmospheric light. The obtained atmosphere light using this method can avoid the influences of noise effectively and has a better stability than Kim et al.'s method.

\section{Extraction Transmission Map}

In order to efficiently overcome the block effect, this paper extracts coarse-scale transmission map using the method based on adaptive clustering, which are described as follows:

Step 1: Cluster the haze images adaptively using Fuzzy C-Means (FCM) .

Step 2: Calculate the minimum channel of each sub-cluster as the initial airlight term Va.

$$
V_{a}(x)=A(1-t(x))=\min _{c \in\{R, G, B\}}\left(\min _{y \in C(x)}\left(I^{c}(y)\right)\right)
$$

where $\mathrm{I}^{\mathrm{C}}$ represents a color channel of haze image I, C(x) denotes the set of pixels from the same sub-cluster with pixel $\mathrm{x}$.

Step 3: Obtain transmission map t1 by substituting the atmospheric light A into Equation (1).

$$
t_{1}=1-V_{a} / A
$$

Since adaptive clustering generates a roughly distribution of the image scene, the obtained transmission reflects the near-far distance of the scene with coarse scale (as shown in Fig. 1(a)).

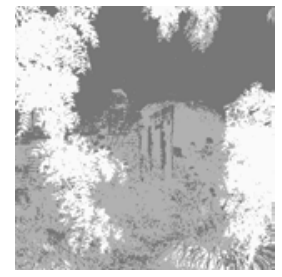

(a)

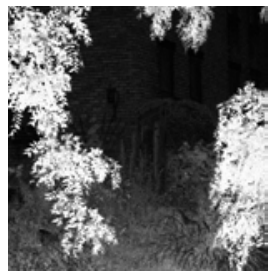

(b)

Fig. 1. The comparisons of transmission (a) The coarse-scale transmission map (b) The fne-scale transmission map

Since HSI color space can separate the luminance component and color information meanwhile it makes people look more natural and intuitive, we convert the image from RGB color space to HSI color space and $\mathrm{S}$ channel is regarded as t2:

$$
t_{2}=1-\frac{3}{R+G+B}[\min (R, G, B)]
$$


where R, G, B represent three channels of the image respectively. Fig. 1(b) shows saturation of the haze image, from which we can see that the saturation can respond the depth information appropriately.

\section{Fusion of Two Scale Transmission Maps}

As can be seen from Fig. 1(a) and (b), transmission map t1 lacks detail depth information while transmission map t2 contains redundant texture information. In order to obtain an accurate transmission map reflecting the depth information truly and naturally, the final transmission map $\mathrm{t}$ is obtained by fusing $\mathrm{t} 1$ and $\mathrm{t} 2$ by using Laplacian pyramid technique.

$$
t_{l}(x)=\sum_{k} L_{l}\left\{t_{k}(x)\right\} \quad k=1,2
$$

where $l$ represents the number of pyramid levels and $L\left\{t_{k}\right\}$ is the Laplacian version of different the transmission maps. This operation is performed successively for each pyramid layer in a bottom-up manner. The final transmission map $\mathrm{t}$ is obtained by summing the fusion results of all pyramid layers as follows:

$$
t(x)=\sum_{l} t_{l}(x) \uparrow^{d}
$$

where $\uparrow^{\mathrm{d}}$ is the upsampling operator with factor $\mathrm{d}=2^{l-1}$. The advantage of Laplacian pyramid technique is that it can generate soft fusion result without sudden jumps.

Once the transmission map $\mathrm{t}$ and atmospheric light $A$ are given, the final haze-free image $J$ can be obtained by the following formula:

$$
J(x)=\frac{I(x)-A}{\max \left(t(x), t_{0}\right)}+A
$$

where $t_{0}$ is a constant. Generally, $t_{0}$ is set to 0.1

\section{Experimental Results and Analysis}

In order to verify the proposed image dehazing algorithm can produce better dehazed results, we compare our method with several state-of-the-art existing single image dehazing methods. Fig. 3 shows the dehazing result comparisons of 'house' and 'mountain' with Fattal et al.'s method [5] and Kim et al.'s method [8]. Obviously, the haze is not removed completely using Fattal et al.'s. Kim et al.'s method cannot effectively remove the haze between the leaves, and even lead to color distortion in local region such as the boundaries of leaves in Fig. 2(c). The areas marked with red rectangles in Fig2. (b-d) exhibit clearly difference. Compared with these methods, the proposed method not only remove the haze completely, but also retain more image detail and color information, meanwhile the visual effect is also very real and natural.

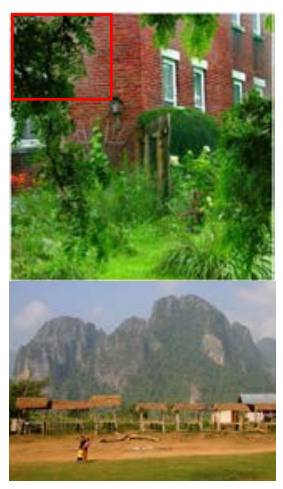

(a)

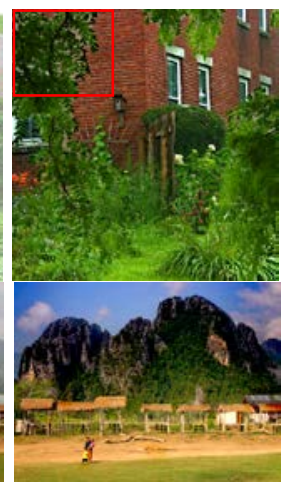

(b)

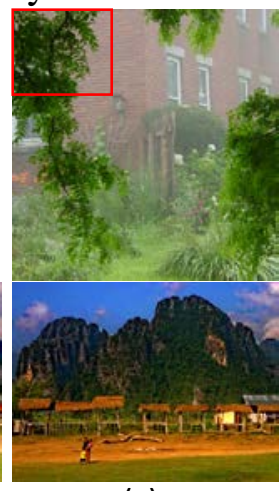

(c)

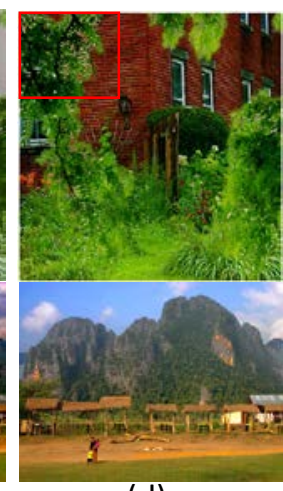

(d)

Fig. 2. The comparisons of 'house' and 'mountain'. (a) The haze image.
(b) Fattal et al.'s result .(c) Kim et al.’s result.
(d) our result.

Through above comparisons and analysis, we can conclude that the proposed image dehazing method can obtain a good and acceptable visual effect. The proposed algorithm is implemented in 
the MATLAB language, and a personal computer with Intel Core(TM) i7-2600 cpu 3.40GHz with 4.0GB RAM is employed in the test. As we all know, although He et al.'s method has good effect for most of the foggy images, which uses the soft matting technique to refine transmission map and is time-consuming. Moreover, exposure is performed as post processing. Therefore, we compare running time of the proposed method with Tarel et al. [7] and Zhang et al. [9] in the same configuration environment, and use the source code provided by the author to calculate the running time for fair comparison. Table 1 shows the comparison results of running time. Obviously, compared with the other two methods, the speed of the proposed method has improved significantly.

Table1. The Comparison of Running Time

\begin{tabular}{lllll}
\hline Image name & Image size & Tarel's/s [7] & Zhang's/s [9] & Ours/s \\
\hline house & $441 \times 450$ & 4.18 & 10.37 & $\mathbf{3 . 9 9}$ \\
mountain & $512 \times 384$ & 4.83 & 11.53 & $\mathbf{2 . 2 2}$ \\
ny17 & $512 \times 384$ & 4.72 & 10.69 & $\mathbf{3 . 8 3}$ \\
Hong Kong & $800 \times 457$ & 18.76 & 21.72 & $\mathbf{6 . 0 4}$ \\
\hline
\end{tabular}

\section{Conclusion and Future Work}

This paper presents an effective single image dehazing method based on fusion technique. First, the coarse-scale transmission map is extracted by the dark prior channel based on adaptive clustering, which can effectively avoid block artifacts caused by mechanical block. Second, we take the saturation of haze image as fine-scale transmission map, which can reflect image depth information sufficiently and naturally. The advantage of this method is straightforward and effective. Moreover, a lot of comparative experimental results indicate that our proposed method can achieve ideal and even better dehazed results.

\section{Acknowledgements}

This work is supported by the Doctoral Science Fund of Liaoning Province (No. 201601349)

\section{References}

[1] Narasimhan S, Nayar S, “Contrast restoration of weather degraded images, ” IEEE PAMI, vol. 25, pp. 713-724, June 2003.

[2] Shwartz S, Namer E, Schechner Y, “Blind haze separation, ” IEEE CVPR, vol. 2, pp. 1984-1991, June 2006.

[3] Kopf J, Neubert B, Chen B, Cohen $M$ et al., "Deep photo: model-based photograph enhancement and viewing,” ACM Trans Graph, vol. 27, pp. 1-10, May 2008.

[4] Tan R, “ Visibility in bad weather from a single image, ” IEEE CVPR, pp. 1-8, June 2008.

[5] Fattal R , “ Single image dehazing, ” ACM Transcations on Graph, vol. 27, pp. 1-9, March 2008

[6] He K, Sun J, Tang X, “ Single image haze removal using dark channel prior, ”Pattern Analysis and Machine Intelligence, vol. 33, pp.2341-2353, December 2011.

[7] Tarel J, Hautière N. “Fast visibility restoration from a single color or gray level image, ”. IEEE ICCV, pp. 2201-2208, Oct. 2009.

[8] Kim J, Jang W, Sim J, Kim C, “Optimized contrast enhancement for real-time image and video dehazing, ” J Vis Commun Image R, vol. 24, Issue 3 pp. 410-425, April 2013.

[9] Zhang Y, Ding Y, Xiao J, Liu J, Guo Z, "Visibility enhancement using an image filtering approach, ”. EURASIP Journal on Advances in Signal Processing, vol. 2012 , No. Issue 220, pp.1-6, January 2012. 